PROCEEDINGS OF THE

AMERICAN MATHEMATICAL SOCIETY

Volume 132, Number 6, Pages 1779-1784

S 0002-9939(03)07256-3

Article electronically published on November 4, 2003

\title{
FREDHOLM ALTERNATIVE AND BOUNDARY VALUE PROBLEMS
}

\author{
STANISŁAW SȨDZIWY \\ (Communicated by Carmen C. Chicone)
}

\begin{abstract}
This note presents a simple proof of A. Lasota's application of the nonlinear Fredholm alternative to the existence proofs of the boundary value problems involving ordinary differential equations. It then uses Lasota's result to get a stronger version of the theorem of Herzog and Lemmert on the Dirichlet boundary value problem for the second-order systems of ordinary differential equations.
\end{abstract}

\section{INTRODUCTION}

It was first observed in Lasota's important papers [6], 7], that the famous Fredholm alternative applies beyond linear problems to cases where the linearity assumption is replaced by conditions of homogeneity. This observation was next used in the investigations of differential and integral equations. (For information concerning the applications of the nonlinear Fredholm alternative, the reader is referred to the monograph [2, or to the more recent papers [9], 8] and their references.)

In [7] the generalized Fredholm alternative was applied to prove the existence of a solution to the boundary value problem (BVP for short)

$$
\begin{gathered}
x^{\prime}(t)=f(t, x(t)), \\
N(x)=r .
\end{gathered}
$$

The solvability of (1.1), (1.2) was deduced from the existence of the unique (trivial) solution to the associated homogeneous BVP for differential inclusion:

$$
\begin{gathered}
x^{\prime}(t) \in F(t, x(t)), \\
N(x)=0 .
\end{gathered}
$$

The purpose of this note is twofold: to give a direct proof of the above result, and to derive from it the more general version of the recent theorem of G. Herzog and R. Lemmert [4] on the solvability of the Dirichlet BVP for the second-order systems of ordinary differential equations. The new proof presented here seems to be natural; it simplifies a rather lengthy reasoning used in [7] which requires Borsuk's theorem (also used in this note) as well as advanced functional analytic tools, such as the Riesz or Banach-Saks theorems.

Received by the editors November 5, 2002 and, in revised form, February 17, 2003.

2000 Mathematics Subject Classification. Primary 34B15.

Key words and phrases. Boundary value problem, Fredholm alternative, Homogeneous map.

(C)2003 American Mathematical Society 


\section{Preliminaries}

(For more information see, e.g., [1.) Denote by $\mathbb{R}^{n}$ the real $n$-space with the Euclidean norm $|\cdot|$. For a subset $G$ of $\mathbb{R}^{n}$ put $d(p, G)=\inf \{|p-q|: q \in G\}$ and let $|G|=\sup \{|q|: q \in G\}$. bdry $D$ and $\operatorname{cl} D$ stand for the boundary and the closure of the set $D$. Set $I=[0,1]$ and let $C(I)$ be the space of continuous functions $x: I \rightarrow \mathbb{R}^{n}$ equipped with the sup norm $|\cdot|_{C}$ and denote $(C(I))^{k}=C(I) \times \cdots \times C(I)$ ( $k$ times).

$\operatorname{cf}\left(\mathbb{R}^{n}\right)$ denotes the set of all nonempty, closed and convex subsets of $\mathbb{R}^{n}$. The Hausdorff distance $\operatorname{dist}(G, H)$ between two closed subsets $G$ and $H \subset \mathbb{R}^{n}$ is defined by the formula $\operatorname{dist}(G, H)=\max \left\{\sup _{q \in G} d(q, H), \sup _{q \in H} d(q, G)\right\}$. The multivalued map $F: \mathbb{R}^{n} \rightarrow \operatorname{cf}\left(\mathbb{R}^{n}\right)$ is said to be continuous if it is continuous with respect to the Hausdorff distance. The map $F: I \rightarrow \operatorname{cf}\left(\mathbb{R}^{n}\right)$ is said to be measurable if for any closed nonempty set $G \subset \mathbb{R}^{n}$ the set $\{t: G \cap F(t) \neq \emptyset\}$ is measurable. Function $F: I \times \mathbb{R}^{n} \rightarrow \operatorname{cf}\left(\mathbb{R}^{n}\right)$ (respectively $f: I \times \mathbb{R}^{n} \rightarrow \mathbb{R}^{n}$ ) is called Carathéodory if for every $t \in I, F(t, \cdot)$ (resp. $f(t, \cdot)$ ) is continuous and for every $p \in \mathbb{R}^{n}, F(\cdot, p)$ (resp. $f(\cdot, p))$ is measurable. Let $C S$ denote the space of Carathéodory functions $f: I \times \mathbb{R}^{n} \rightarrow \mathbb{R}^{n}$ such that for any compact $K \subset \mathbb{R}^{n}$ there is a function $\psi_{K}$, summable on $I$, satisfying $|f(t, p)| \leq \psi_{K}(t)$ for $(t, p) \in I \times K$.

The function $x: I \rightarrow \mathbb{R}^{n}$ is called a solution of (1.3) (resp. (1.1)) provided that $x$ is absolutely continuous and satisfies (1.3) (resp. (1.1)) for almost all $t \in I$.

\section{Results}

The theorem given below generalizes the Fredholm alternative, since the maps $N$ and $F$, which appear in its formulation, need not be linear.

Theorem 1 (cf. [7] Théorème 2.1]). Let $f: I \times \mathbb{R}^{n} \rightarrow \mathbb{R}^{n}, F: I \times \mathbb{R}^{n} \rightarrow \operatorname{cf}\left(\mathbb{R}^{n}\right)$ be the Carathéodory functions and let $N: C(I) \rightarrow \mathbb{R}^{n}$ be continuous. Assume that:

(A) $F$ and $N$ are homogeneous (i.e., for all $t \in I,(a, p) \in \mathbb{R} \times \mathbb{R}^{n}, F(t, \lambda p)=$ $\lambda F(t, p), N(\lambda x)=\lambda N(x)$ for all $(\lambda, x) \in \mathbb{R} \times C(I)$ ) and the function $\varphi(t)=$ $\sup _{|p|=1}|F(t, p)|$ is summable on $I$,

(B) $f$ satisfies condition

$$
\lim _{n \rightarrow \infty}(1 / n) \int_{I} \sup _{|p| \leq n} d(f(t, p), F(t, p)) d t=0 .
$$

(C) $x(t) \equiv 0$ is the unique solution of the homogeneous BVP (1.3), (1.4).

Then for any $r \in \mathbb{R}^{n}$ the BVP (1.1), (1.2) has at least one solution.

If $(\mathrm{A})$ and $(\mathrm{C})$ hold and the conditions imposed on $N$ and $f$ appearing in (A) and $(\mathrm{B})$ are replaced by

(D) the map $N$ is linear and continuous, and the function $f$ satisfies the conditions

$$
f(t, q)-f(t, p) \in F(t, q-p), \quad \forall q, p \in \mathbb{R}^{n} \quad \int_{I}|f(t, 0)| d t<\infty,
$$

then for any $r \in \mathbb{R}^{n}$ the solution to BVP (1.1), (1.2) exists and is unique.

As a corollary to Theorem 1, we get an extension of the result of G. Herzog and R. Lemmert.

Theorem 2 ([4, Theorem 1]). Assume that the map $N_{1}:(C(I))^{2} \rightarrow \mathbb{R}^{2 n}$ is continuous and homogeneous. Let $h:[0,1] \times \mathbb{R}^{2 n} \rightarrow \mathbb{R}^{n}$ be an asymptotically linear 
Carathéodory function, i.e.,

$$
\lim _{n \rightarrow \infty} \int_{I} \sup _{|x|+|v| \leq n} \frac{|h(t, x, v)-A(t) x-B(t) v|}{|x|+|v|} d t=0,
$$

where $A, B: I \rightarrow \mathbb{R}^{n \times n}$ are matrix functions with summable entries.

If $w(t) \equiv 0$ is the only solution of the homogeneous $B V P$

$$
w^{\prime \prime}=A(t) w+B(t) w^{\prime}, \quad N_{1}\left(w, w^{\prime}\right)=0,
$$

then for each $r_{0} \in \mathbb{R}^{2 n}$, the $B V P$

$$
\begin{gathered}
u^{\prime \prime}(t)=h\left(t, u(t), u^{\prime}(t)\right), \\
N_{1}\left(u, u^{\prime}\right)=r_{0}
\end{gathered}
$$

is solvable.

Remark. Theorem 2 has been proved in 4] for $N_{1}\left(w, w^{\prime}\right)=(w(0), w(1))$, assuming that $h, A, B$ are continuous and satisfy the condition

$$
\lim _{|x|+|v| \rightarrow \infty} \frac{|h(\cdot, x, v)-A(\cdot) x-B(\cdot) v|_{C}}{|x|+|v|}=0,
$$

obviously more restrictive than (3.3). Theorem 2 extends in a natural way to the case of BVPs for systems of $2 k$-th order asymptotically linear differential equations with boundary conditions of the form $N\left(u, u^{\prime}, \ldots, u^{(k-1)}\right)=r$, where $r \in \mathbb{R}^{n k}$ and $N:(C(I))^{k} \rightarrow \mathbb{R}^{n k}$ is continuous and homogeneous.

As claimed in 4], for $n=1$, the conditions for the solvability of BVP (3.4), (3.5) have been given by A. I. Perov (see [5]), but the techniques used in the scalar case are not applicable for $n>1$. Thus G. Herzog and R. Lemmert investigate the problem with the shooting method. It is worth noting (without providing details) that a straightforward proof of the existence result for BVP (3.4),(3.5) with $N_{1}$ linear can be obtained using the Green function approach in a way similar to the one described in [3, Ch. XIII, Part II].

\section{Proofs}

The proof of Theorem 1, presented below, is elementary since, besides Borsuk's odd map theorem, it does not require any advanced functional analytic results.

Set $Z=C(I) \times \mathbb{R}^{n}$ and for $z=(y, q) \in Z$ let $|z|_{Z}=|y|_{C}+|q|$. For functions $g, e: I \times \mathbb{R}^{n} \rightarrow \mathbb{R}^{n}$ and $r \in \mathbb{R}^{n}$ define maps $T(g), R(e, r): Z \rightarrow Z$ by

$$
\begin{aligned}
T(g)(z) & =\left(\int_{0}^{t} g(s, y(s)) d s+q, N(y)+q\right), \\
R(e, r)(z) & =\left(\int_{0}^{t} e(s, y(s)) d s,-r\right) .
\end{aligned}
$$

Observe that if $N$ is continuous and $g, e \in C S$, then the maps $T$ and $R$ are completely continuous.

Lemma. Let $F: I \times \mathbb{R}^{n} \rightarrow c f \mathbb{R}^{n}, g: I \times \mathbb{R}^{n} \rightarrow \mathbb{R}^{n}$ be Carathéodory functions and let $g(t, x) \in F(t, x)$ for all $(t, x) \in I \times \mathbb{R}^{n}$. Let $T(g), R(e, r)$ be defined by (4.1).

If $(\mathrm{A})$ and $(\mathrm{C})$ hold, then the homogeneous equation

$$
u=T(g)(u)
$$


has only the trivial solution, and for all $(e, r) \in C S \times \mathbb{R}^{n}$, the solutions to the equation

$$
u=T(g)(u)+R(e, r)(u)
$$

satisfy the inequality $|u|_{Z} \leq \gamma|R(e, r)(u)|_{Z}$ with a constant $\gamma>0$ independent of $(e, r)$.

Proof of Lemma. The first part of the statement follows from (C), since from $g(t, x)$ $\in F(t, x)$ it follows that (4.2) is equivalent to BVP (1.3), (1.4).

To show the second part of the statement, suppose that there is a sequence $\left\{u_{n}\right\}=\left\{\left(x_{n}, r_{n}\right)\right\} \subset Z$ such that

$$
u_{n}=T(g)\left(u_{n}\right)+R\left(e, r_{n}\right)\left(u_{n}\right),
$$

and $\left|u_{n}\right|_{Z} \geq n\left|R\left(e, r_{n}\right)\left(u_{n}\right)\right|_{Z}$.

Set $v_{n}=\left(y_{n}, q_{n}\right)=u_{n} /\left|u_{n}\right|_{z}$. Then $\left|v_{n}\right|_{z}=1$ and the sequence $\left\{v_{n}\right\}$ contains a convergent subsequence $\left\{v_{k(n)}\right\}=\left\{\left(y_{k(n)}, q_{k(n)}\right)\right\}$. The convergence of $\left\{q_{k(n)}\right\}$ is obvious. To prove the existence of a convergent subsequence of $\left\{y_{n}\right\}$, note that $\left\{y_{n}(t)\right\}$ is uniformly bounded, and by (4.3),

$$
v_{n}=\left(1 /\left|u_{n}\right|_{Z}\right)\left(T(g)\left(u_{n}\right)+R\left(e, r_{n}\right)\left(u_{n}\right)\right) .
$$

It follows from (A) that $\left(1 /\left|u_{n}\right|_{Z}\right)\left(g\left(s, x_{n}(s)\right) \in \lambda F\left(s, x_{n}(s) /\left|x_{n}\right|_{C}\right)\right.$, where $\lambda=$ $\left|x_{n}\right|_{C} /\left|u_{n}\right|_{Z} \leq 1$; hence $\left(1 /\left|u_{n}\right| z\right) \mid\left(g\left(s, x_{n}(s)\right) \mid \leq \varphi(s)\right.$, which together with the inequality

$$
\left|R\left(e, r_{n}\right)\left(u_{n}\right)\right|_{Z} /\left|u_{n}\right|_{Z} \leq(1 / n)
$$

shows that the sequence $\left\{y_{n}\right\}$ is equicontinuous and by the Ascoli theorem, it contains a convergent subsequence $\left\{y_{k(n)}\right\}$. Consequently, setting $\lim _{n \rightarrow \infty} v_{k(n)}=$ $v_{0}$, from (4.4) and (4.5), one gets $v_{0}=T(g)\left(v_{0}\right)$, contradicting (4.2) because $\left|v_{0}\right|_{Z}=1$.

Proof of Theorem 1. For $(t, p) \in I \times \mathbb{R}^{n}$ define $h(t, p) \in F(t, p)$ by

$$
d(f(t, p), F(t, p))=|f(t, p)-h(t, p)| .
$$

Function $h(t, p)$ is well defined because $F(t, p) \in \operatorname{cf}\left(\mathbb{R}^{n}\right)$ and the space $\left(\mathbb{R}^{n},|\cdot|\right)$ is strictly convex. $h$ is a Carathéodory function, which follows from the fact that both functions $f$ and $F$ are Carathéodory (see, e.g., [1, Cor. 8.2.13]).

Denote $k(t, p)=f(t, p)-h(t, p)$ and define the maps $T(h)$ and $R(k, r)$ by (4.1) setting $g=h, e=k$ and put $S(f)=T(h)+R(k, r)$. By (3.1) and (A), $f, h \in C S$; so the map $S(f)$ is completely continuous.

Since the solutions to BVP (1.1), (1.2) are fixed points of $S(f)$, the proof reduces to showing that $S(f)(w)=w$ for a certain $w \in Z$. To this end we show that there is an open, bounded and symmetric subset $D$ of $Z$, satisfying $0 \in D$ and such that

$$
\begin{gathered}
S(f)(z) \neq z \text { for } z \in \text { bdry } D \\
\alpha(z-S(f)(z)) \neq(1-\alpha)(-z-S(f)(-z)) \text { for } \alpha \in[1 / 2,1], z \in \text { bdry } D .
\end{gathered}
$$

By Borsuk's theorem [10, Ch. III], this will imply that $S(f)$ has a fixed point in $D$.

Fix $r \in \mathbb{R}^{n}$. Applying (3.1), choose an $a>0$ to satisfy $\max \left\{|R(k, r)(z)|_{Z}:|z|_{Z}=\right.$ $a\}<a / \gamma$, with $\gamma$ defined as in the lemma. We prove that the set $D=\left\{z:|z|_{Z}<a\right\}$ has the properties required in (4.6).

Suppose, if possible, that $u=S(f)(u)$ and $|u|_{Z}=a$. Then, by the lemma, $|u|_{Z} \leq \gamma|R(k, r)(u)|_{Z}<\gamma(a / \gamma)=a$ and we get a contradiction; so the first inequality (4.6) holds. 
For the proof of the second inequality, assume that there are $z_{0}=\left(y_{0}, q_{0}\right)$ and $\beta$ satisfying $\left|z_{0}\right|_{Z}=a, \beta \in[1 / 2,1]$ and

$$
z_{0}=\beta(T(h)+R(k, r))\left(z_{0}\right)-(1-\beta)(T(h)+R(k, r))\left(-z_{0}\right) .
$$

By $(\mathrm{A}), h\left(s,-y_{0}(s)\right) \in-F\left(s, y_{0}(s)\right)$. Hence from the convexity of $F\left(s, y_{0}(s)\right)$, it follows that $e\left(s, y_{0}(s)\right)=\beta h\left(s, y_{0}(s)\right)-(1-\beta) h\left(s,-y_{0}(s)\right) \in F\left(s, y_{0}(s)\right)$. Moreover, the homogeneity of $N$ implies that $\beta N\left(y_{0}\right)-(1-\beta) N\left(-y_{0}\right)=N\left(y_{0}\right)$ and as a consequence of $(4.1)$ we get $\left(\beta T(h)\left(z_{0}\right)-(1-\beta) T(h)\left(-z_{0}\right)=T(e)\left(z_{0}\right)\right.$. Thus (4.7) assumes the form

$$
z_{0}=T(e)\left(z_{0}\right)+\beta R(k, r)\left(z_{0}\right)-(1-\beta) R(k, r)\left(-z_{0}\right) .
$$

Since $\left|\beta R(k, r)\left(z_{0}\right)-(1-\beta) R(k, r)\left(-z_{0}\right)\right|_{Z} \leq \max \left\{|R(k, r)(z)|_{Z}:|z|_{Z}=a\right\}<a \gamma$, from the lemma applied to the equation above it follows that $\left|z_{0}\right|_{Z}<\gamma(a / \gamma)=a$, which gives a contradiction and proves the second formula (4.6). The reference to Borsuk's theorem completes the first part of Theorem 1.

The proof of the remaining part repeats the argument of [7]: Condition (D) implies that $f$ and $N$ satisfy (A) and (B); hence by the first part of Theorem 1, BVP (1.1), (1.2) has a solution. Its uniqueness follows in an obvious way from (C) and the first formula of (3.2).

Proof of Theorem 2. BVP (3.4), (3.5) is equivalent to the BVP of the form (1.1), (1.2), where: $f(t, x)=P(t) x+g(t, x), N(x)=N_{1}\left(u, u^{\prime}\right), x \in \mathbb{R}^{2 n}$ has components $x_{1}, x_{2} \in \mathbb{R}^{n}$,

$$
P(t)=\left(\begin{array}{cc}
0 & I \\
A(t) & B(t)
\end{array}\right), \quad g(t, x)=\left(\begin{array}{c}
0 \\
\varphi(t, x)
\end{array}\right), \quad x_{1}=u
$$

$I$ is an $n \times n$ unit matrix and $\varphi(t, x)=h\left(t, x_{1}, x_{2}\right)-A(t) x_{1}-B(t) x_{2}$.

Now the proof reduces to observing that the (single-valued) map $F$ defined by $F(t, x)=\{P(t) x\}$ and the maps $g, N$ defined above satisfy all the assumptions of Theorem 1 .

\section{REFERENCES}

[1] J. P. Aubin and H. Frankowska, Set-Valued Analysis, Birkhäuser, Boston, Basel, Berlin, 1990. MR 91d:49001

[2] S. Fučik, J. Nečas, and V. Souček, Spectral Analysis of Nonlinear Operators, Lecture Notes in Mathematics, Vol. 346, Springer-Verlag, Berlin, Heidelberg, New York, 1973. MR 57:7280

[3] P. Hartman, Ordinary Differential Equations, John Wiley and Sons, New York, 1964. MR 30:1270

[4] Gerd Herzog and Roland Lemmert, An existence theorem for systems of boundary value problems, Proc. Amer. Math. Soc. 128(1) (2000), 157-160. MR 2000c:34046

[5] M. A. Krasnoselski, A. I. Perov, A. I. Povolozki, and P. P. Sarbiejko, Vektorfelder in der Ebene, Akademie-Verlag, Berlin, 1966. MR 34:1995

[6] A. Lasota, Sur une généralisation du premier théorème de Fredholm, Bull. Acad. Polon. Sci., Sér. Sci. Math., Astronom. Phys. XI (1963), 89-94. MR 27:6154

[7] A. Lasota, Une généralisation du premier théorème de Fredholm et ses applications à la théorie des équations différentielles ordinaires, Annales Polonici Mathematici XVIII (1966), 65-77. MR 33:2849

[8] R. Manasévich and P. Takač, On the Fredholm alternative for the p-Laplacian in one dimension, Proc. London Math. Soc. (3) 84 no. 2 (2002), 324-342. MR 2003b:34045 
[9] B. Ruf, A nonlinear Fredholm alternative for second order ordinary differential equations, Math. Nachr. 127 (1986), 299-308. MR 88f:34027

[10] J. T. Schwartz, Nonlinear Functional Analysis, Gordon and Breach, New York, 1969. MR 39:2014

Institute of Computer Science, Jagiellonian University, Ul. Nawojki 11, 30-072 CraCOW, POLAND

E-mail address: sedziwy@softlab.ii.uj.edu.pl 